prick test.Also peripheral blood samples were obtained to determine serum levels of IgE and blood eosinophil cell count in both study groups.

Results A total of 52 patients with psoriasis, and 50 healthy subjects as the control group were considered. Between allergic diseases only asthma was lower in psoriatic patients rather than controlbased on ISAAC questionarie $(p=0.044)$. There is no significant diffrence between result of skin prick test (atopy) in case and control group(p). Also no significant correlation was found between eosinophil count $(\mathrm{p}=0.057)$ and Ig E level in case and control group $(p=0.88)$.

Conclusions In general, these findings suggest that the Asthma was not common in psoriasis, and support the concept that Asthma protects against the autoimmune disease such as psoriasis.

\section{ASSOCIATED FACTORS ON SLEEP DISORDERS IN SYSTEMIC LUPUS ERYTHEMATOSUS}

${ }^{1} Y$ Li. ${ }^{1}$ Xiangya hospital - Central South University, Department of Rheumatology and Immunology, Chang Sha, China

\subsection{6/lupus-2017-000215.251}

Background and aims To investigate the prevalence of sleep disorders and the relevant determinants in a cohort of SLE patients.

Methods One hundred patients with SLE were included in the study. Sleep quality was assessed using the Pittsburgh Sleep Quality Index (PSQI). Depression, anxiety, quality of life, and fatigue were evaluated by PHQ-9, GAD-7, SF-36, and VAS respectively. The demographic and clinical data were also recorded. SLE disease activity and damage severity were assessed by SLEDAI and SDI respectively.

Results The prevalence of sleep disorders in SLE patients was $42 \%$. Compared with patients without sleep disorders, the ratio of males and married patients, age, the score of SDI, PHQ-9, GAD-7, and fatigue were higher in SLE patients with sleep disorders, while the score of SF-36 was lower $(p<0.05)$. Age, SLEDAI, SDI, PHQ-9, GAD-7, and fatigue correlated positively with sleep disorders $(p<0.05)$. C3 and the score of SF-36 correlated negatively with sleep disorders $(\mathrm{p}<0.05)$. In multiple logistic regression analyses, gender, anxiety, body pain, and energy were the independent determinants of sleep disorders $(\mathrm{R} 2=0.494, \mathrm{P}<0.01)$.

Conclusions Poor sleep quality is common in SLE patients. Gender, age, disease activity and severity, anxiety, depressed mood, and quality of life contribute significantly to sleep disorders in SLE.

\section{THE LONG-TERM OUTCOMES ON LEFLUNOMIDE TREATMENT IN PATIENTS WITH LUPUS NEPHRITIS: A TWELVE-YEAR FOLLOW-UP STUDY}

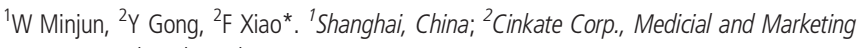
Department, Shanghai, China

\subsection{6/lupus-2017-000215.252}

Background and aims One phase III study has reported that Leflunomide(LEF) in combination with prednisone was effective in the induction therapy of proliferative lupus nephritis (LN). However, long-term data of LEF in the maintenance treatment of $\mathrm{LN}$ are lacking. The aim of this study was to investigate the long-term outcomes of LEF in the maintenance treatment of LN in an open-label extension of a prospective multi-centre observational study.

Methods 70 patients were enrolled into LEF induction group with a loading dose of $50 \mathrm{mg} /$ day for 3 days, followed by $30 \mathrm{mg} /$ day for six months. The partial and complete remission rate was $52 \%$ and $21 \%$. Among them, 15 patients voluntarily entered an open labelled extension study. Data for survival and kidney function were prospectively collected during a 12 year period for the 15 patients who were maintained on $20 \mathrm{mg} /$ day LEF.

Results 15 patients (mean age $40.2 \pm 7.4$ years; $86.6 \%$ female) were treated with LEF for $>12$ months, the mean duration of LEF treatment was 11.8 years. During a median follow-up period of 12.3 years, 1 patient died from sepsis, 4 patients developed renal flare. The 12 year cumulative survival rates for the composite end point of death and chronic renal failure were $93.3 \%$ and $100 \%$, respectively. At the final follow-up, the complete remission rate in the remaining patients was $71.43 \%$, the partial remission rate was $28.57 \%$, the mean proteinuria leval was $0.33 \mathrm{~g} /$ day(range $0.07-1.62 \mathrm{~g} /$ day), and the mean serum creatinine leval was $62.3 \mathrm{umol} / \mathrm{L}$.

Conclusions Our data suggest that a remission-inducing regimen of LEF followed by maintenance therapy with LEF resulted in good long-term patient survival and renal preservation.

\section{A COMPARISON OF OVARIAN RESERVE IN WOMEN WITH SYSTEMIC LUPUS ERYTHEMATOSUS FOLLOWING TREATMENT WITH INTRAVENOUS CYCLOPHOSPHAMIDE VIS ORAL MYCOPHENOLATE MOFETIL}

${ }^{1} \mathrm{P}$ Potturi*, ${ }^{1} \mathrm{~S}$ Sharma, ${ }^{2} \mathrm{P}$ Sikka, ${ }^{3} \mathrm{M}$ Rathi, ${ }^{4} \mathrm{~N}$ Sachdeva, ${ }^{1} \mathrm{~S}$ Jain. ${ }^{1} \mathrm{PGIMER}$, Internal Medicine, Chandigarh, India; ${ }^{2} P G I M E R$, Gynaecology and Obstetrics, Chandigarh, India: ${ }^{3}$ PGIMER, Nephrology, Chandigarh, India; ${ }^{4}$ PGIMER, Endocrinology, Chandigarh, India

\subsection{6/lupus-2017-000215.253}

Introduction Immunosuppression with Cyclophosphamide is treatment of choice in lupus nephritis. Ovarian reserve depletion is well known complication. Early identification of depletion of ovarian reserve can be done by correlating antral follicle count with AMH.

Aims- To assess the ovarian reserve in patients who have receive d IV Cyclophosphamide vs those who have received Mycophenolate-mofetil for SLE by measuring $\mathrm{AMH}$, Inhibin $\mathrm{B}, \mathrm{FSH}$ and antral follicular count.

Methods A prospective Case-Control study with 50 patients who were diagnosed with SLE were studied. Twenty-five cases and controls defined by females age 18-40 with SLE were enrolled. Cases received IV CYC as per NIH protocol and controls received oral MMF. Baseline FSH, LH, E2, AMH, Inhibin and antral follicular count, and at 6 months were done in both the groups.

Results Mean age: cases- 31.36 \pm 6.357 , controls- $28.36 \pm 4.396$ (p-0.058).

Conclusions MMF did not cause decrease in ovarian reserve. IV CYC caused Subclinical depletion of ovarian reserve with significant decreased in $\mathrm{AMH}$, Inhibin B and E2 levels and follicular counts. AMH and AFC are good markers to assess subclinical depletion of ovarian reserve 